\title{
Machine-learning integrated glassy defect from intricate configurational-thermodynamic-dynamic space
}

\author{
Zeng-Yu Yang, ${ }^{1,2}$ Dan Wei, ${ }^{1,2}$ Alessio Zaccone, ${ }^{3,4,5}$ and Yun-Jiang Wang ${ }^{1,2, *}$ \\ ${ }^{1}$ State Key Laboratory of Nonlinear Mechanics, Institute of Mechanics, \\ Chinese Academy of Sciences, Beijing 100190, China \\ ${ }^{2}$ School of Engineering Science, University of Chinese Academy of Sciences, Beijing 100049, China \\ ${ }^{3}$ Department of Physics "A. Pontremoli", University of Milan, via Celoria 16, Milan 20133, Italy \\ ${ }^{4}$ Department of Chemical Engineering and Biotechnology, \\ University of Cambridge, Cambridge CB3 $0 A S$, UK \\ ${ }^{5}$ Cavendish Laboratory, University of Cambridge, Cambridge CB3 OHE, UK
}

(Dated: July 30, 2021)

\begin{abstract}
Optimizing materials' properties and functions by controlling defects in the crystalline phase has been a cornerstone of materials science and condensed matter physics. However, this paradigm has yet to be established in the broadly defined amorphous materials, which implies identification of very subtle structural features in an otherwise uniformly disordered medium. Here we propose and define a new integrated glassy defect (IGD), based on machine learning strategy informed by atomistic physics, and also by an extremely wide configurational, thermodynamic, and dynamic variables space of the disordered state. The IGD simultaneously includes positional topology and vibrational features, as well as the local morphology of the potential energy landscape. This unprecedented combination gives rise to a much more comprehensive and more effective definition of the "glassy defect", much beyond the conventional, purely structural input. IGD can be used not only as an efficient predictor of athermal plasticity, but is also transferable to detect both short-time vibrational anomalies (the boson peak), and long-time relaxation and diffusion dynamics in glasses. The integrated strategy is instrumental to build the long-sought structure-property relationship in complex media.
\end{abstract}

\section{INTRODUCTION}

Owing to plenty of attractive mechanical, physical and chemical properties and a wide range of potential structural and functional applications, the metallic glasses (MGs) are both of engineering significance and great scientific relevance for the broad communities of materials science and condensed matter physics [1-5]. Despite decades of relentless pursuit, establishment of the structure-property relationship in such disordered materials is still one of the most elusive yet most intriguing unsolved problems up to date [6-13]. In contrast with their crystalline counterparts, whose structural defects are easily identified from the periodic arrangement of atoms in the lattice, the atomic-scale structure of amorphous materials is usually featureless. The structure of glass lacks either translational or rotational periodicity, with each atom residing in its own, individual atomic environment. Thus it is rather difficult to find a physics-motivated descriptor, like the Burgers' vector of dislocation in crystal, that is capable of characterizing the possible structural defects in glasses, which act as plasticity-carriers under external mechanical and/or thermal stimuli [14]. The lack of a priori structural defects thus prevents establishing an intuitive one-to-one structure-property relationship in the widespread domain of amorphous materials.

Great efforts have been devoted to identifying possible structure-property relationships in the disordered

*yjwang@imech.ac.cn materials over the past decades. Among these attempts, the most successful representatives include the mean-field free volume [15] and shear transformation zone (STZ) models [16-18]. However, no structural defects that could play the same role as dislocations in crystals, have been reported in the literature about glassy materials [19]. Until recent years, a few effective structural descriptors have been used to explain the observed dynamics of amorphous materials. Such structural signatures can be categorized into three groups. The first category is based on purely static structure, such as the shortrange Voronoi polyhedra [7, 20] and the related local fivefold symmetry parameter [21, 22], as well as the Voronoi cell anisotropy [23, 24], the inversion symmetry breaking [25], the two-body excess entropy [26, 27], the local coordination number [28], and other structural order metrics [29-31], etc. The second class involves descriptors of thermodynamic response, e.g. soft vibrational modes [32-34], vibrational mean-squared displacement (vMSD) or Debye-Waller factor [35, 36], the flexibility volume $[37,38]$, and finally the local thermal energy [39]. The third group contains further dynamic features, which includes the local yield stress [40], the gradient along the minimum energy pathway of STZ on the potential energy landscape (PEL) [41], etc. However, these physicsmotivated parameters usually partially forecast the nature of the localized plastic flow in glasses. And often the prediction is either material-specific, or deformationprotocol dependent [41].

Instead of the conventional knowledge-driven strategy, the new machine learning (ML) paradigm is especially 
suitable for constructing a robust correlation between structures and functions in glasses [8, 42-48]. It has been set in practice by combining a series of particleposition based symmetric Gaussian functions, and by exploring interstice structural distributions in both short and medium range via ML models. In all the pioneering works, the machine-learned structural defects, e.g. the glass "softness" [43], the "quench-in softness" [45], and the "structural flexibility" [48] are strongly correlated with the elementary excitation in different glasses. However, the protocols are only based on purely static structure. The lack of high-order features may cause incomplete description of the dynamics in glass. For example, strong spatio-temporal correlation has been found between structure and dynamics in model glasses $[29,31]$, which suggests opening up a new avenue of research, where an overarching structure-property "superrelationship" can be developed based on even broader physical input and not just the structural features. Actually, the recent concept of the flexibility volume [37] goes in this direction as it combines both structural (Voronoi volume) and thermodynamic (vibrational) feature of a specific atom to predict dynamics.

Therefore, a novel proposition about a structureproperty super-relationship must simultaneously account for all (structural, thermodynamic, dynamic) physical aspects that are associated with properties of glass. To this end, we propose a machine-learning integrated glassy defect (IGD) of MGs via extraction of all configurational, thermodynamic and dynamic features that are coupled and coexisting in the inherent configuration of disordered phase. The extensive ML process is conducted by using atomic coordinates, vibrational analysis and the PEL morphology, respectively, as different but equally important fingerprints of the underlying glass physics. The integrated defect via ML - termed integrated glassy defect (IGD) - serves as an unprecedented signature of the athermal local structural deformation (plasticity), as well as thermal short-time vibrational anomalies, long-time diffusion and slow relaxation dynamics in the amorphous alloys.

\section{METHODOLOGY}

\section{A. Molecular dynamics}

All of the molecular dynamics (MD) simulations here are performed by using the open source LAMMPS code [49], with the Finnis-Sinclair-type embedded-atom method empirical potential [50] being adopted to describe the interatomic interaction of the $\mathrm{Cu}_{50} \mathrm{Zr}_{50}$ metallic glasses. We prepare 10 independent glass samples and each of them contains 19652 atoms, with 3D dimensions of $\sim 70 \times 70 \times 70 \AA$. For the glass structure preparation, the samples are first melted from their crystalline phases ( $\mathrm{B}_{2}$ phase, body-centered cubic) from $0 \mathrm{~K}$ to $2000 \mathrm{~K}$, and then being equilibrated for $2 \mathrm{~ns}$ at $2000 \mathrm{~K}$ before

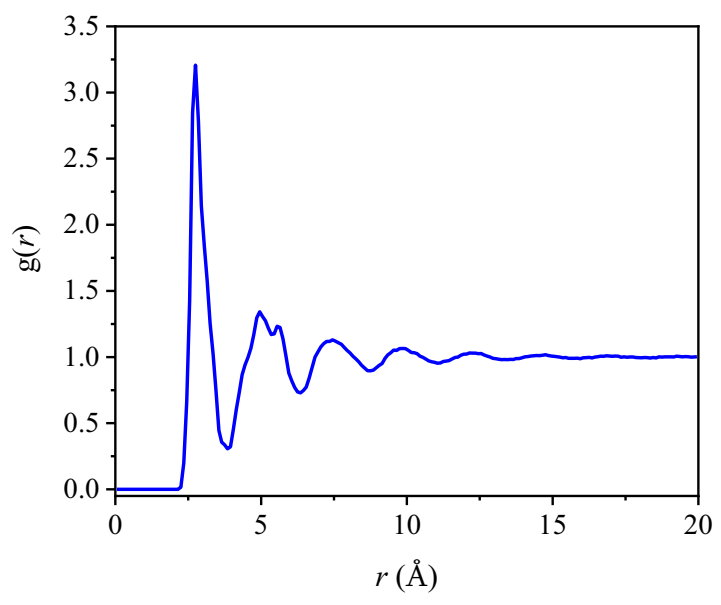

FIG. 1. The radial distribution function of a $\mathrm{Cu}_{50} \mathrm{Zr}_{50}$ metallic glass. The split of the second peak in RDF indicates glass nature of the atomic model.

being quenched to the glassy state at $0 \mathrm{~K}$, with a cooling rate of $10^{10} \mathrm{~K} / \mathrm{s}$. Periodic boundary conditions (PBCs) and NPT ensemble (constant number of atoms, constant pressure, and constant temperature) are applied for all the atomistic simulations. Temperature is controlled by the Nosé-Hoover thermostat [51]. Stress tensor is kept zero within the Parrinello-Rahman barostat [52]. The MD time step is 2 fs to numerically integrate the Newton's equation of motion. Fig. 1 gives the radial distribution function (RDF) of one $\mathrm{Cu}_{50} \mathrm{Zr}_{50}$ sample. It clearly indicates the nature of glass structure by the split of second peak.

Athermal quasi-static shear (AQS) simulations [53] with simple $x y, y z$, and $z x$ shearing deformation protocols are performed at a step size of $\Delta \gamma=1 \times 10^{-5}$. Each deformation increment is followed by operation of energy minimization to remove all the thermal effect. In this way, the strain rate effect on the mechanical response is avoided in the shear deformation and, thus, reflecting intrinsic nature of the local plastic event carried out by the atomic rearrangement - shear transformation.

\section{B. Construction of the structural-thermodynamic-dynamic space}

\section{Structural features}

We adopt a family of symmetric Gaussian functions to describe the information embedded in the structural space. The structural descriptor $G-i$ for each atom, which has been used to calculate the "softness" [43] and details the potential energy landscape for complex materials [42], is defined as

$$
G_{i}=\sum_{j}^{N_{i}} \exp \left[-\left(R_{i j}-\mu\right)^{2} / L^{2}\right] .
$$


Here $R_{i j}$ denotes the distance between atom $i$ and $j . N_{i}$ is the number of the neighbouring atoms around the $i$ th atom. $\mu$ and $L$ are constants that need to be tested and optimized. Actually, the Gaussian structural function is a weighted sum of a group of the Gaussian functions. Parameter $\mu$ controls the position of the highest weight and the parameter $L$ denotes the speed of the weight drop. According to the $\mathrm{RDF}$ of the present $\mathrm{Cu}_{50} \mathrm{Zr}_{50}$ model glass as shown in Fig. 1, the parameter $\mu$ is set from 1.5 to $7.5 \AA$ with an interval of $0.2 \AA$. It makes sure that all the effective structural information from short-range to medium-range order is contained in the ML model. Moreover, the parameter $L$ is set as $3.5 \AA$ for each Gaussian structural function. Therefore, there are altogether 31 Gaussian structural functions included in the machine learning model. Gaussian functions at longer distance has been tested but make trivial contribution to the effectiveness of IGD and therefore not considered in the ML model.

A new purely static structure feature proposed here is the two-body excess entropy at single-particle level, which naturally encompasses positional information and their correlation in space. It is defined as the two-body term of the infinite-term expansion of the excess entropy, which represents the loss of configurational entropy with respect to the ideal gas state owing to the positional correlation in some ordered phase [26]. This two-body approximation has been applied to account for the structure-dynamic relationship of the glass-forming liquids [54]. Therefore, it is certainly an intriguing pure positional feature that can enrich the information of the structural space in the machine learning model. The twobody excess entropy, or the local structural entropy, of the $i$ th atom is calculated according to the equation

$$
\begin{gathered}
S_{2, i}=-\frac{1}{2} \sum_{v} \rho_{v} \int_{0}^{r_{\text {cutoff }}}\left\{g_{i}^{u v}(\mathbf{r}) \ln g_{i}^{u v}(\mathbf{r})-\right. \\
\left.\left[g_{i}^{u v}(\mathbf{r})-1\right]\right\} d \mathbf{r} .
\end{gathered}
$$

Here $u$ and $v$ represent the types of particles $(\mathrm{Cu}$ or $\mathrm{Zr}$ atoms in the present case), $\rho_{v}$ is the number density of the $v$ type particles, and $g_{i}^{u v}$ denotes the local RDF between the particle $i$ of type $u$ and other particles of type $v$. The cutoff distance for the integration is set from 3 to $15 \AA$ with step of $0.5 \AA$. Furthermore, the freedom of choice in the cutoff distance provides immense room to construct the structural spaces for machine learning. On the basis of two-body entropy, another 25 structural features are augmented to the Gaussian functions.

\section{Thermodynamic features}

To detail the thermodynamic information in a quantitative manner, the vibrational mean-squared displacement (vMSD), or the Debye-Waller factor, is defined according to the equation:

$$
\left\langle\Delta r_{i}^{2}\right\rangle=\left\langle\left|\mathbf{r}_{i}(t)-\mathbf{r}_{i, \text { equil }}\right|^{2}\right\rangle_{\tau}
$$

Here $\mathbf{r}_{i}(t)$ is the instantaneous position at different times during tracing for $\tau=100 \mathrm{ps}$ at different temperatures, from 25 to $500 \mathrm{~K}$ with an interval of $25 \mathrm{~K}$. $\mathbf{r}_{i \text {,equil }}$ is the equilibrium position of the $i$ th atom in the inherent structure after energy minimization. The angular brackets imply the time average. This appropriate time duration ensures that the atoms vibrate around their thermodynamic equilibrium positions without dynamic hopping from one energy basin to another. Thus we obtain 20 features to construct the thermodynamic space preparing for the machine-learning model. The wide temperature range enables inclusion of possible anharmonic nature of the local energy basin.

\section{Dynamic features}

To assign a series of meaningful dynamic properties to the atoms in glass, the activation-relaxation technique nouveau (ARTn) $[55,56]$ is adopted to extract the singleatom activation energies. In the framework of ARTn, the "activation" is initiated by imposing a random small displacement to the local cluster centered on a chosen atom. In the present work, the perturbation is restricted to a specific atom by setting the cutoff distance to be $2 \AA$, which is shorter than the first maximum of RDF, $g(r)$, of the $\mathrm{Cu}_{50} \mathrm{Zr}_{50}$ glass, as shown in Fig. 1. The activation direction for a possible event is chosen randomly, with magnitude of the perturbation displacement being fixed $0.1 \AA$. Then the activation is driven along the minimum Hessian direction. The increment movement is $0.15 \AA$. When the lowest eigenvalue of Hessian matrix is less than $-0.30 \mathrm{eV} / \AA^{2}$, the system is pushed towards a connecting saddle point by using the Lanczos algorithm. During the "relaxation" process, the maximum force tolerance of $0.05 \mathrm{eV} / \AA$ is used for converging to the saddle point. As shown in Fig. 2e, the wide distribution of energy barriers for an atom indicates the complexity, or multiplicity, of the activation paths on the PEL. Therefore, single activation energy of an atom is not enough to detail the local topology of PEL where initial state resides. For statistical purpose, each atom is furthermore activated for 24 times with random initial perturbation direction. Consequently, 24 activation energies for each atom is arranged in order according to their magnitude as $\left\{\Delta Q_{1}, \Delta Q_{2}, \ldots, \Delta Q_{24}\right\}$, where $\Delta Q_{1}<\Delta Q_{2}<\ldots<\Delta Q_{24}$. Finally, we adopt such arrangement to detail the distribution of activation energies for an atom. We therefore obtain 24 features accounting for the dynamic information of an atom by including both difficulty (the barrier height) and multiplicity (possible paths) of the local structural excitations.

The distributions of activation energies for all the atoms, $\mathrm{Cu}$ atoms and $\mathrm{Zr}$ atoms are shown in Fig. 2(a)(c), respectively. It shows that the activation energy is distributed over a very broad range, indicating a strong dynamic heterogeneity as frequently reported in the literature, whereas the distribution for the $\mathrm{Cu}$ atoms is more 


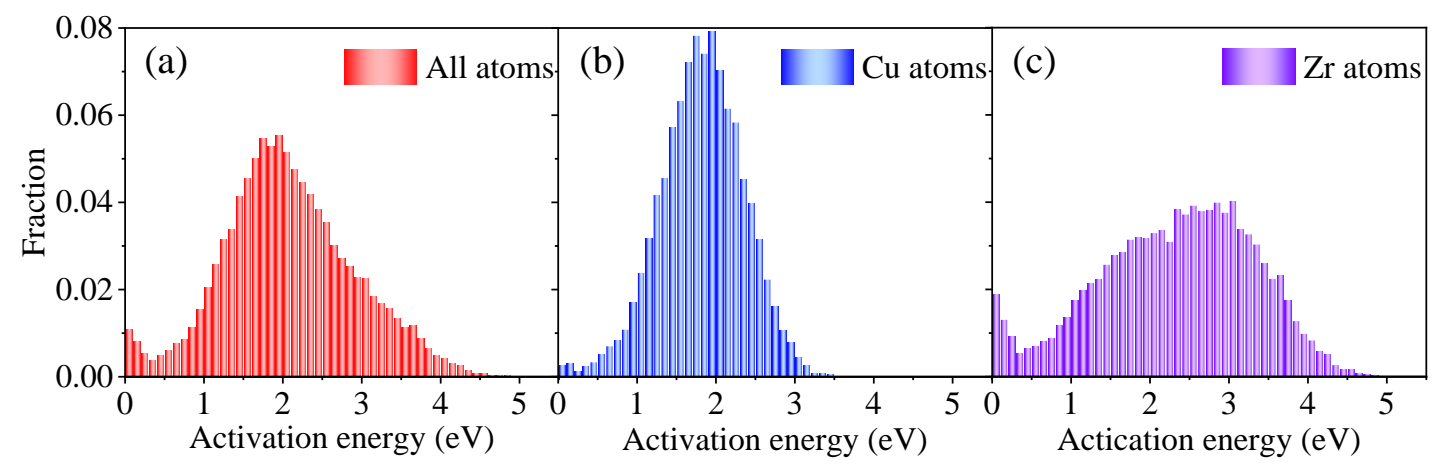

(d)

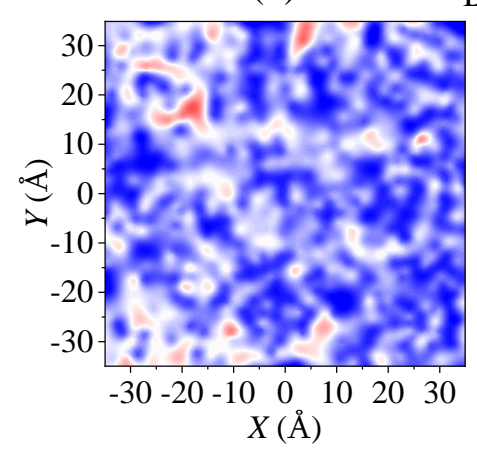

Barrier (eV)

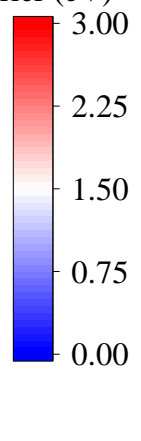

(e)

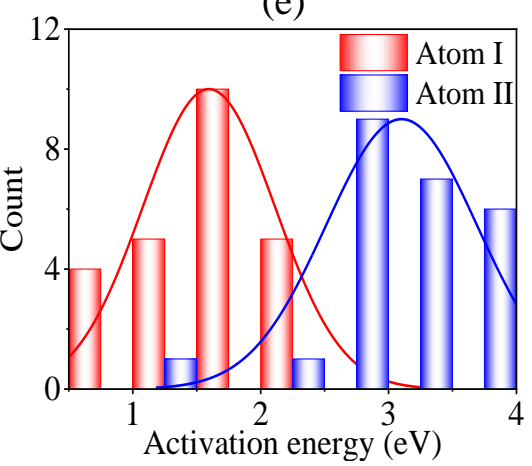

FIG. 2. Dynamic features in $\mathrm{Cu}_{50} \mathrm{Zr}_{50}$ metallic glass shown by potential energy landscape. The distribution of activation energies for (a) all atoms, (b) Cu atoms and (c) Zr atoms, respectively. (d) Color map of the spatial distribution of atomic activation energies. The shown slice is normal to the $z$ direction with a thickness of $5 \AA$. (e) The wide distribution of activation barriers for two specific atoms. Each atom has been activated for 24 times along random directions.

concentrated than that of the $\mathrm{Zr}$ atoms. This is because the coordination number of $\mathrm{Zr}$ atoms is higher than that of $\mathrm{Cu}$ atoms, resulting in a greater diversity in the local structural environment of Zr atoms. Compared with $\mathrm{Cu}$ atoms, the peak position of the $\mathrm{Zr}$ barrier distribution shifts to the right. This is due to that $\mathrm{Zr}$ atoms are heavier than $\mathrm{Cu}$ atoms, and the former are difficult to be activated under the same external conditions. In other words, compared with $\mathrm{Cu}$ atoms, $\mathrm{Zr}$ atoms require a longer time to relax into stable positions corresponding to the deeper basin of PEL. This phenomenon may be the result of the larger constraints that $\mathrm{Zr}$ atoms undertake in $\mathrm{CuZr}$ glass than $\mathrm{Cu}$ atoms.

In Fig. 2(d), we show the spatial distribution of $\Delta Q_{1}$ in a slice chosen on the $x-y$ plane of the model. This plot shows the topological information on activation energy and indicates the spatial heterogeneity of the PEL in glass.

\section{Supervisory signal for classification - shear transformation}

To identify the atoms that have undergone structural rearrangement, we use the non-affine squared displacement $D_{\min }^{2}$ [17] and monitor its jump as global strain evolves. To calculate the parameter, the first step is to optimize a local affine transformation matrix, $\mathbf{J}_{i}$, that best maps $\left\{\mathbf{d}_{j i}^{0}\right\} \rightarrow\left\{\mathbf{d}_{j i}\right\}, \forall j \in N_{i}$, where $N_{i}$ is the number of the nearest neighbors of the $i$ th atom, and $\mathbf{d}_{j i}^{0}$ and $\mathbf{d}_{j i}$ are the bond vectors for the reference and the current configurations between the $j$ th and $i$ th (central) atoms, respectively. The local non-affine squared displacement of the central atom $i$ relative to its nearest neighbor atoms $j$, is then defined as

$$
D_{i, \text { min }}^{2}=\frac{1}{N_{i}} \sum_{j}\left(\mathbf{d}_{j i}-\mathbf{J}_{i} \mathbf{d}_{j i}^{0}\right)^{2} \text {. }
$$

In the present simulations, we monitor a flow reference $\Delta D_{\min }^{2}$ to compare two configurations that are close by to one another, i.e. consecutive configurations separated by a global shear strain difference of $\Delta \gamma=0.01 \%$ under AQS. A typical example is shown in Fig. 3(a), where $\Delta D_{\text {min }}^{2}$ of specific atoms is plotted as a function of shear strain up to $\gamma=5 \%$. Atom I and II can be observed to undertake obvious jump at about $\gamma=1.6 \%$ and $\gamma=1.8 \%$, respectively, whereas atom III exhibits no jump seen by $\Delta D_{\min }^{2}$. Fig. 3(b) shows the distribution of the maximum $\Delta D_{\min }^{2}$ of the atoms. Here, we chose the turning point of $0.05 \AA^{2}$ as the threshold to judge whether atoms are taken to be the ones that have undertaken rearrangement or not. For each sample (as we have 10 samples), we perform AQS along $x y, y z$, and $z x$ directions, respectively. The $\Delta D_{\text {min }}^{2}$ for each atom in the three AQS loading protocols is monitored and, once the value of $\Delta D_{\min }^{2}$ exceeds the threshold of $0.05 \AA^{2}$, the 

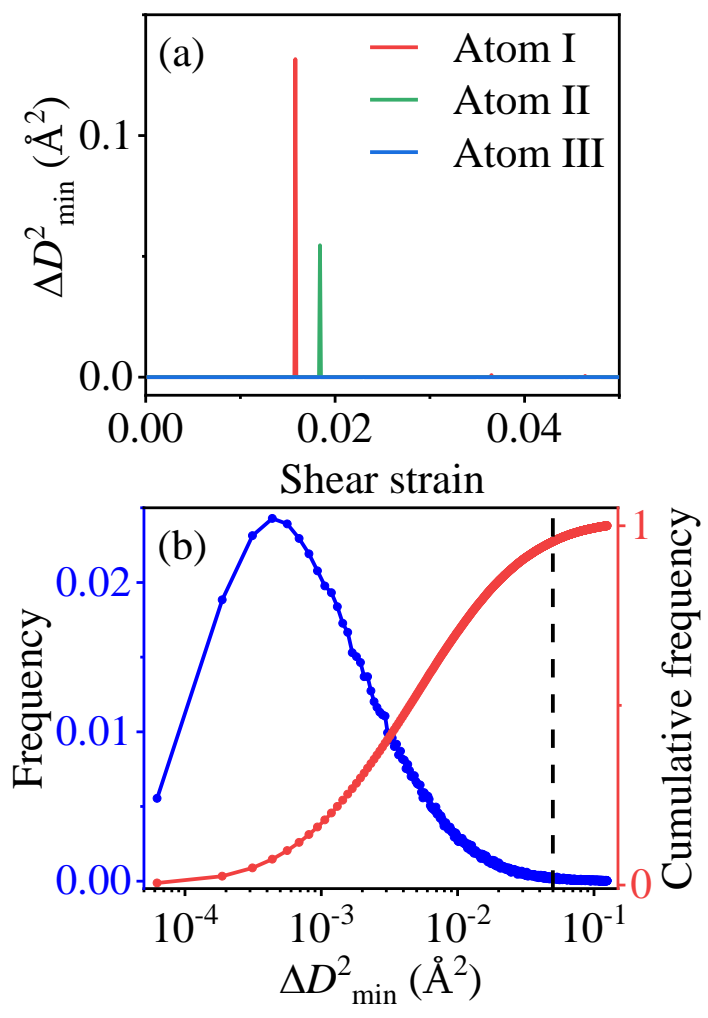

FIG. 3. (a) $\Delta D_{\min }^{2}$ as a function of the global shear strain for three specific atoms in $\mathrm{Cu}_{50} \mathrm{Zr}_{50}$ under AQS to a shear strain $\gamma=0.05 . \Delta D_{\min }^{2}$ evaluates the non-affine displacement between two configurations that are close by to one another with shear strain difference of $\gamma=0.01 \%$. Atom I and II have apparently experienced obvious jumps in $\Delta D_{\min }^{2}$ (and likely local structural excitation) but not atom III. (b) Distribution of the maximum jump of $\Delta D_{\min }^{2}$ with the blue line denoting the frequency for different $\Delta D_{\min }^{2}$, whereas the red line characterizing the cumulative frequency.

corresponding atom will be labeled as a rearranged atom. As shown in Fig. 3(b), where the position of the threshold value is marked using dashed line, the rearranged particles contain about $5 \%$ of all atoms.

\section{Support vector machine model}

The training set is given as the form of $\left\{\left(\mathbf{x}_{1}, y_{1}\right), \ldots,\left(\mathbf{x}_{N}, y_{N}\right)\right\}$, where $N$ denotes the number of atoms and $\mathbf{x}_{i}=\left\{x_{i}^{1}, \ldots, x_{i}^{M}\right\}^{\mathrm{T}}$ describes the feature space of atom $i$, with $M$ denoting the number of features. In the present work, $M=100$. Here, $y_{i}=1$ if the $i$ th atom have undergone obvious shear transformation, while $y_{i}=-1$ for the atoms that are marked as non-rearranged ones. The aim of our SVM model is to find a hyperplane in the high-dimensional feature space that can separate data points with different value of $y_{i}$ as preciously as possible. For this purpose, the SVM algorithm of the LIBSVM package [57] is used

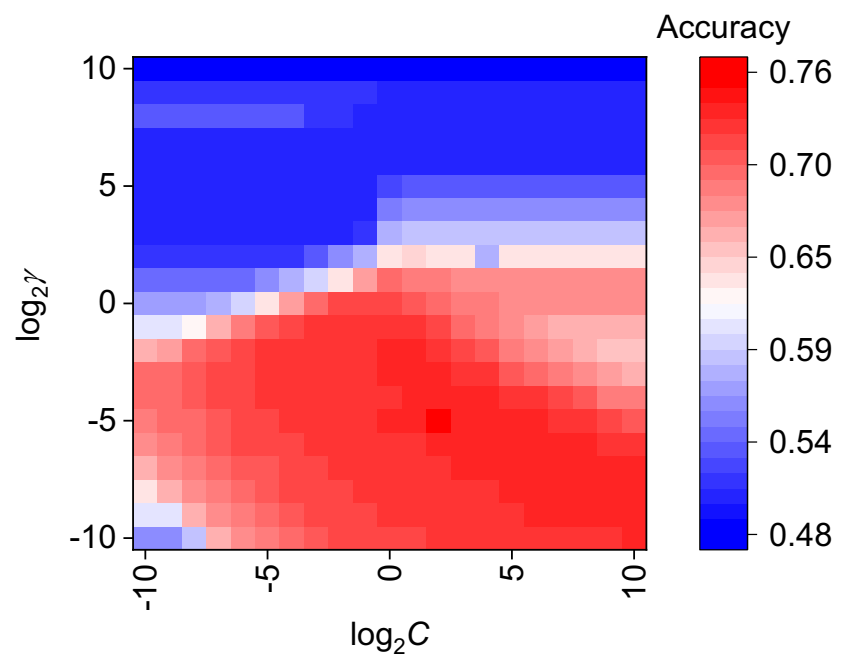

FIG. 4. Contour plot of cross-validation accuracy. The RBF (Gaussian) kernel function is used and predictions from models with different parameters are shown. The $x$-axis is $\log _{2} C$ and the $y$-axis is $\log _{2} \gamma$. The best model is identified to be the one with the largest cross-validation accuracy. And thus parameters are set as $C=4$ and $\gamma=0.03125$.

to solve the following primal optimization problem.

$$
\begin{array}{r}
\min _{\boldsymbol{\omega}, \xi, b} \frac{1}{2} \boldsymbol{\omega}^{T} \boldsymbol{\omega}+C \sum_{i=1}^{N} \xi_{i} \\
\text { subject to } \mathrm{y}_{\mathrm{i}}\left(\boldsymbol{\omega}^{\mathrm{T}} \phi\left(\mathbf{x}_{\mathrm{i}}\right)+\mathrm{b}\right) \geq 1-\xi_{\mathrm{i}} \\
\xi_{i} \geq 0, i=1, \ldots, N,
\end{array}
$$

Here, $\phi\left(\mathbf{x}_{i}\right)$ maps $\mathbf{x}_{i}$ into a higher-dimensional space, $\boldsymbol{\omega}$ is the vector variable used to construct the hyperplane, and $C>0$ is the regularization parameter. Due to the high dimensionality of $\boldsymbol{\omega}$, this dual problem is always solved by defining the kernel function as $K\left(\mathbf{x}_{i}, \mathbf{x}_{j}\right)=\phi\left(\mathbf{x}_{i}\right) \cdot \phi\left(\mathbf{x}_{j}\right)$. In the present work, the radial basis function (RBF, Gaussian) kernels with the form of $K\left(\mathbf{x}_{i}, \mathbf{x}_{j}\right)=\exp \left\{-\gamma\left\|\mathbf{x}_{i}-\mathbf{x}_{j}\right\|^{2}\right\}$ are adopted. So $(C, \gamma)$ are the flexible parameters to be decided. We provide a possible interval of $C$ and $\gamma$ in the grid space. The tunable parameters are then optimized through crossvalidation in order to obtain the highest accuracy. The heat map of cross-validation accuracy for different $(C, \gamma)$ parameters is shown in Fig. 4. It indicates that the crossvalidation accuracy is found to have a maximum value when $C=2^{2}$ and $\gamma=2^{-5}$. These values are therefore used in the present SVM model for classification.

\section{E. Short-time vibrational anomaly - boson peak}

To test the correlation between IGD and the shorttime thermal properties of metallic glass, we propose and implement a single-atom level intensity of boson peak, which is the excess vibrational mode in the low frequency 

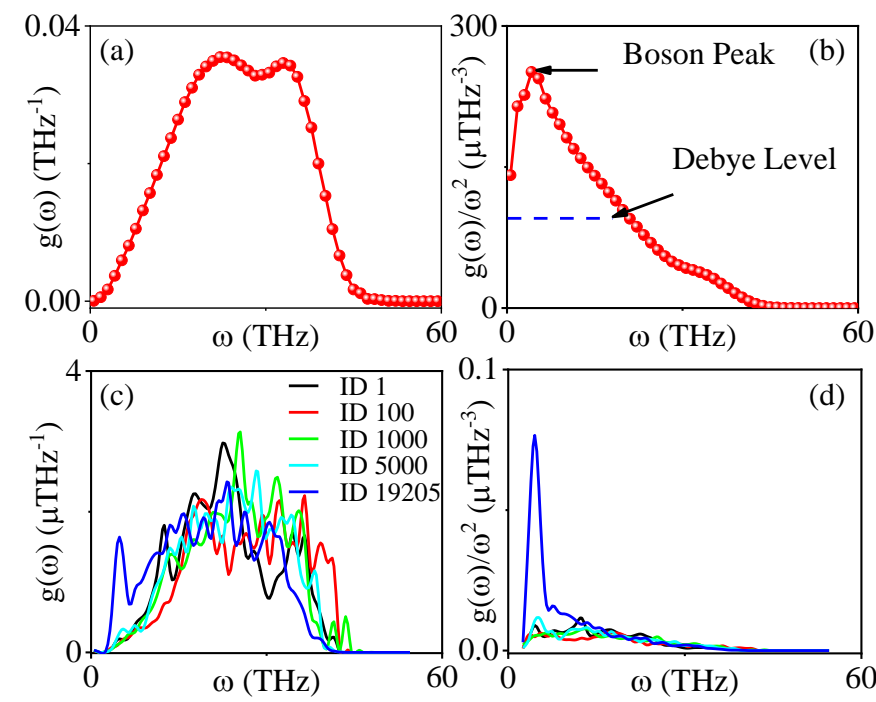

FIG. 5. Phonon features for the $\mathrm{Cu}_{50} \mathrm{Zr}_{50}$ metallic glass. (a) The VDOS for the system. (b) The reduced VDOS $g(\omega) / \omega^{2}$ for the system. It confirms the existence of boson peak in $\mathrm{Cu}_{50} \mathrm{Zr}_{50}$ with the dashed line identifying the Debye level. (c) VDOS for several specific atoms. (d) The reduced VDOS $g(\omega) / \omega^{2}$ or intensity of boson peak for several different atoms.

domain of phonons in glass, compared with the Debye squared model. First of all, the vibrational, or phonon, density of states (VDOS) for the system are calculated by direct diagonalization of the Hessian matrix, which is evaluated at a local energy minimum position of the potential energy landscape - an inherent structure. The Hessian matrix are constructed by evaluating the spring constants of each atom from the empirical potential. The single-atom VDOS of the atom $i$ is then formulated as [58],

$$
g_{i}(\omega)=\frac{1}{3 N} \sum_{j} \delta\left(\omega-\omega_{j}\right)\left|\mathbf{e}_{j}^{i}\right|^{2}
$$

Here, $N$ is the number of atoms in the system. $\omega_{j}$ and $\mathbf{e}_{j}$ give the normal model frequency and the corresponding polarization vector of the vibrational mode $j$, respectively. Compared with the Debye squared model, i.e. $g(\omega) \propto \omega^{2}$, the intensity of the single-particle boson peak $I_{\mathrm{B} P}$ is obtained by finding the maximum value of the reduced VDOS, i.e. $I_{\mathrm{BP}}=\max \left[g_{i}(\omega) / \omega^{2}\right]$.

Figure 5(a) shows the VDOS of $\mathrm{Cu}_{50} \mathrm{Zr}_{50}$ metallic glass. In Fig. 5(b), we show the reduced VDOS by the Debye-squared model, $g(\omega) \sim \omega^{2}$ (the VDOS divided by $\left.\omega^{2}\right)$. It indicates the existence of a boson peak in the system and shows that the boson peak can be clarified at around $5 \mathrm{THz}$. Fig. 5(c) shows the calculated singleparticle VDOS for several atoms and the corresponding boson peaks for the specific atoms are shown in Fig. 5(d).
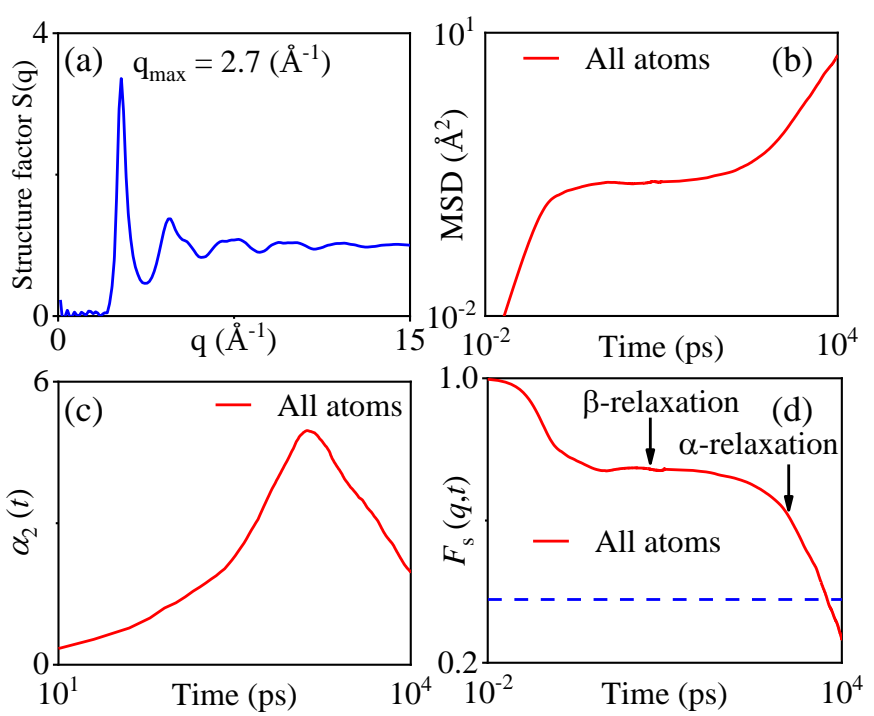

FIG. 6. Long-time dynamics for $\mathrm{Cu}_{50} \mathrm{Zr}_{50}$ metallic glass. (a) The static structure factor of $\mathrm{Cu}_{50} \mathrm{Zr}_{50}$. (b) The meansquared displacement at $700 \mathrm{~K}$ for all atoms in the system. (c) The non-Gaussian parameter at $700 \mathrm{~K}$ for all atoms. (d) Self-intermediate scattering function with wave vector $q=2.7$ $\AA^{-1}$ and temperature $T=700 \mathrm{~K}$ for all atoms.

\section{F. Long-time dynamics - diffusion and relaxation}

\section{Diffusive mean-squared displacement}

A commonly used long-time diffusive feature of amorphous solids is the mean-squared displacement (MSD), which quantifies how far a particle has moved with respective to its initial position as a function of time, i.e.

$$
\Delta r_{i}^{2}(t)=\left\langle\left[\mathbf{r}_{i}(t)-\mathbf{r}_{i}(0)\right]^{2}\right\rangle
$$

Here $\mathbf{r}_{i}(t)$ and $\mathbf{r}_{i}(0)$ denote the positions of the particle $i$ at the current and the initial configuration, respectively. The angle bracket $\langle\cdots\rangle$ represents an ensemble average. Here MSD is calculated at $700 \mathrm{~K}$ for the $\mathrm{Cu}_{50} \mathrm{Zr}_{50}$ sample. Note that the diffusive MSD is different from the vibrational MSD as shown in Eq. (3), the latter describes a thermodynamic response.

The diffusive mean-squared displacement as a function of time at $700 \mathrm{~K}$ for $\mathrm{Cu}_{50} \mathrm{Zr}_{50}$ sample is shown in Fig. 6(b). It indicates that MSD in metallic glass shows three stages. At very short timescales, particle motion is free from the influence of the neighboring atoms and therefore show ballistic behavior with MSD is proportional to $t^{2}$. As the timescale increases, neighboring atoms function as obstacle and block the further movement of the central atom. Therefore, topological excitation for particles become difficult and there is certainly a plateau shown Fig. 6(b). Eventually, atoms will break away from the bondage of the surrounding atoms and undergo random walk diffusive motion. 


\section{Non-Gaussian parameter}

The non-Gaussian parameter is commonly used to measure the dynamical heterogeneity of a disordered system. While the diffusive MSD $\left[\Delta r_{i}^{2}(t)\right]$ is related to the second order of the local atom position, the non-Gaussian parameter is calculated by the fourth order of the particle's position. It is defined as

$$
\alpha_{2}(t)=\frac{3\left\langle\sum_{i}^{N}\left[\mathbf{r}_{i}(t)-\mathbf{r}_{i}(0)\right]^{4}\right\rangle}{5\left\langle\sum_{i}^{N}\left[\mathbf{r}_{i}(t)-\mathbf{r}_{i}(0)\right]^{2}\right\rangle^{2}}-1 .
$$

Here $N$ is the number of atoms in the system. For Brownian motion, the atomic displacement follows Gaussian distribution. The non-Gaussian parameter therefore characterizes the degree of deviation from the Gaussian distribution of particle motion. Thus, $\alpha_{2}(t)=0$ for Brownian motion. For the present metallic glass, the distribution of atomic displacements becomes non-Gaussian at intermediate times as shown in Fig. 6(c). This is due to the development of balance between the mobile and immobile atoms in the system. Therefore, $\alpha_{2}(t)$ is always positive at any time. The maximum value of $\alpha_{2}(t)$ measures the heterogeneity of diffusion, and the time when $\alpha_{2}^{\max }(t)$ appeared indicates the duration of heterogeneity establishment.

\section{Self-intermediate scattering function and the $\alpha$-relaxation time}

To understand the feature of relaxation dynamics, we investigate the self-intermediate scattering function (SISF) and the $\alpha$ relaxation time of the present $\mathrm{Cu}_{50} \mathrm{Zr}_{50}$ metallic glass. The generalized form of SISF is defined as

$$
F_{\mathrm{S}}(q, t)=\frac{1}{N} \sum_{l}^{N}\left\langle\exp \left\{-i \mathbf{q} \cdot\left[\mathbf{r}_{l}(t)-\mathbf{r}_{l}(0)\right]\right\}\right\rangle,
$$

where $N$ is the number of atoms, $\mathbf{q}$ is the wave-vector corresponding to the main peak of the static structure factor $S(q)$. In the present work, the wave-vector is chosen $2.7 \AA^{-1}$ as shown in Fig. 6(a). The evolution of SISF is similar to that of the diffusive MSD. Fig. 6d shows the SISF of the whole $\mathrm{Cu}_{50} \mathrm{Zr}_{50}$ glass sample as a function of time. It shows a feature of two-step relaxation, i.e. the short-time $\beta$-relaxation and the long-time $\alpha$-relaxation. The $\alpha$-relaxation time $\tau_{\alpha}$ is thereafter defined as the timescale when SISF decays to $e^{-1}$, as labeled by a horizontal dashed line in Fig. 6(d).

\section{RESULTS}

\section{A. Framework of machine learning}

Our goal is to develop a supervisory model, using the atomistically-informed machine learning, to simultaneously include the structural, thermodynamic, and dynamic aspects of the glass physics. Crucially the model should be capable of distinguishing "fertile" atoms that are responsible for the plastic rearrangements from those "sessile" atoms that are not.

Figure 7 shows the workflow illustrating the classification process via machine learning. The first step consists of collecting the dataset: we simulate a binary $\mathrm{Cu}_{50} \mathrm{Zr}_{50}$ system and 10 independent glass configurations are prepared by a rapid cooling method, with each sample containing 19652 atoms. This composition has been shown to be the most challenging one for predictions of glassy and plastic behavior, owing to a lower degree of both structural and dynamic heterogeneity compared to other stoichiometries [48]. Therefore, it provides an upper threshold for predictability. But this choice, on the other hand, represents the best ground to demonstrate the validity of the ML strategy. As shown in Fig. 7, there are three categories of the input features. First, the radial symmetry Gaussian functions and the twobody excess entropy are used to characterize the static structural features of each atom. These quantities naturally include both radial and structural-order information about the glassy structures. Second, the vMSD, or the Debye-Waller factor, at different temperature is utilized to characterize the thermodynamic fingerprints of each atom. Deviations from the harmonic approximation (higher-order thermal response) become prominent with increasing temperature. Third, a series of meaningful dynamic properties can be captured by the local topologies of the PEL. A series of activation energies provide information about steepness (or height) and multiplicity (or width) of the possible shear transformation pathways. A recent work [59] pointed out the importance of the PEL topology, and has included the arithmetic mean energy barrier as the supervisory signal of ML to predict $\beta$ relaxation in a metallic glass from purely static structure. In contrast, here we employ a hierarchically-sorted list of activation energies of possible structural excitations as the learning descriptors relevant to the topology of PEL. All together, we have built an abundant configurationalthermodynamic-dynamic phase space of glass for ML classification. The couplings between the different features have been considered. Specifically, we construct a dataset consisting of 56 structural (31 Gaussian functions and 25 two-body entropies), 20 thermodynamic, and 24 dynamic descriptors for each atom, respectively. Consequently, the feature vector reads $\mathbf{x}_{i}=\left\{x_{i}^{1}, \cdots x_{i}^{M}\right\}^{\mathrm{T}}=$ $\left\{G_{i}\left(r_{1}\right), \cdots G_{i}\left(r_{31}\right) ; S_{2, i}\left(r_{1}\right), \cdots S_{2, i}\left(r_{25}\right) ; \Delta r_{i}^{2}\left(T_{1}\right)\right.$, $\left.\cdots \Delta r_{i}^{2}\left(T_{20}\right) ; \Delta Q_{1}, \cdots \Delta Q_{24}\right\}^{\mathrm{T}}$ with $M=100$.

To identify an essential structural rearrangement, AQS deformation [53] is conducted up to a global shear strain 


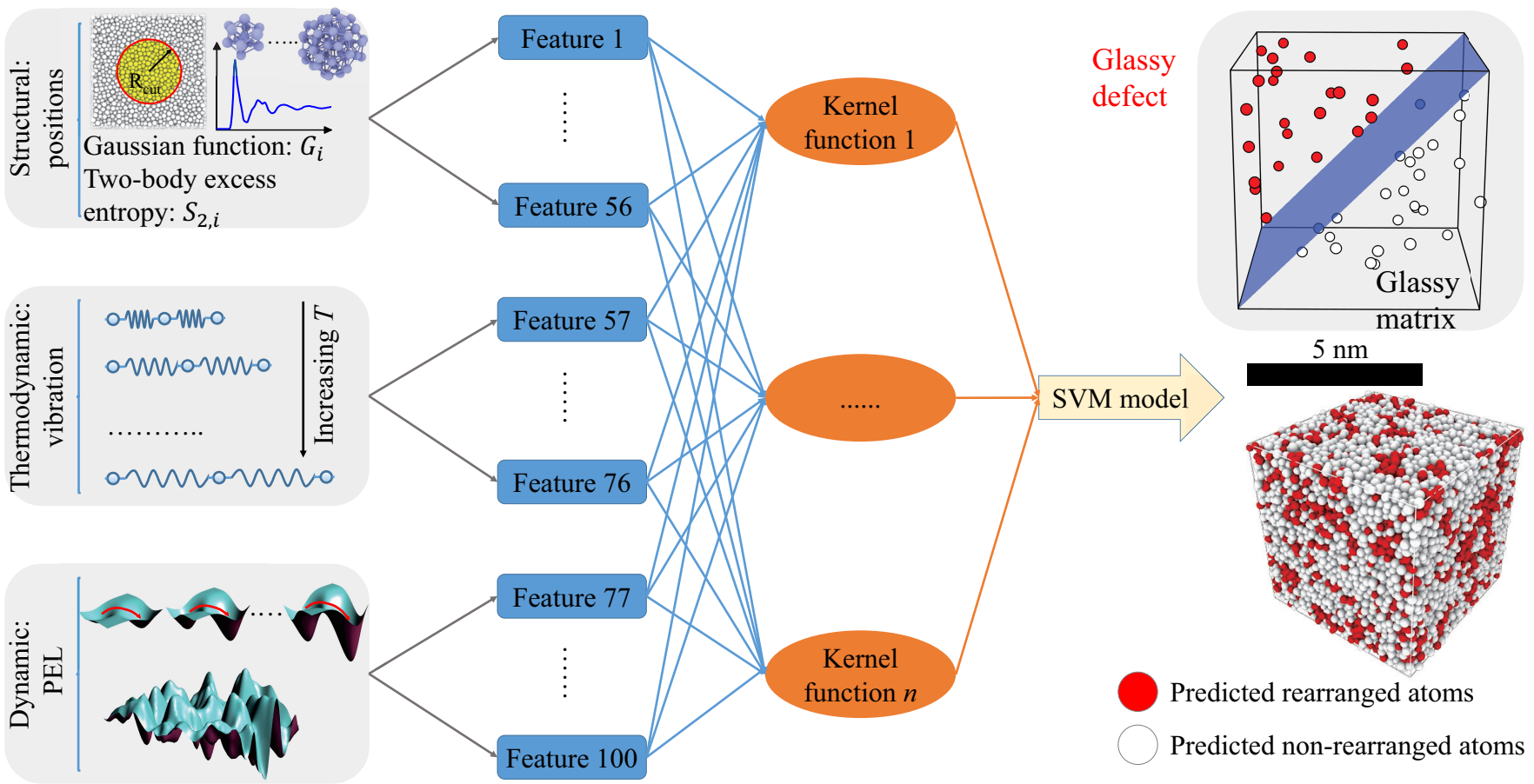

FIG. 7. Machine-learning encoding of the integrated glassy defect. A fingerprint vector of each atom $-\mathbf{x}_{i}-$ is constructed from the combined configurational, thermodynamic, and dynamic spaces. Then, all the descriptors are trained by the SVM model, which classifies the atoms to be either structural defect or glassy matrix, with the non-affine atomic strains as the supervisory signal.

magnitude of 0.05 . We track possible STZ events by monitoring the instantaneous jump in the non-affine displacement $D_{i, \min }^{2}$ [17], which plays the role of a supervisory signal in local plastic events. The next task is to select, at random, a subset of atoms as the training set and label the atoms with an index either $y_{i}=-1$ or 1 . As shown in Fig. 3(b), an atom $i$ is labeled as $y_{i}=1$ if it rearranges within $\Delta D_{i, \text { min }}^{2}$ is higher than $0.05 \AA$, and $y_{i}=-1$ if it does not. Approximately, $5 \%$ of all atoms have undergone structural rearrangements after AQS loading up to $5 \%$. The training set is constructed by selecting all the atoms that have undergone rearrangements $(\sim 10000$ atoms), and an equal number of atoms that do not experience rearrangement in the first nine samples (among total 10 samples). In this work, we train our machinelearning architecture using the support-vector machine (SVM) scheme, which builds a hyperplane in the multidimensional space to best separate the data points with different labels. Once this hyperplane is established for the training set, the tenth sample is treated as the testing set for calibration.

\section{B. Predicting plastic atoms via machine learning}

Figure 8 illustrates the capability of the proposed ML model in identifying whether an atom is susceptible to plastic rearrangement (or equivalently, belongs to flow defects) or not. Fig. 8(a) visualizes the spatial distri-
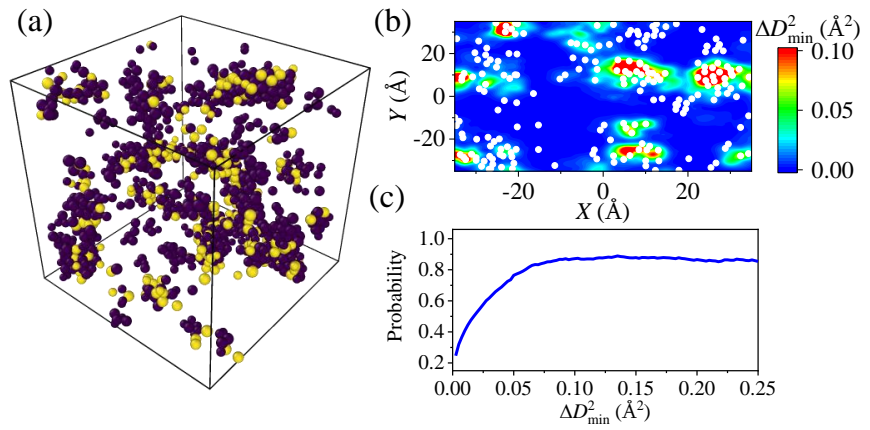

FIG. 8. Prediction of athermal excitation. (a) Spatial distribution of the atoms that have undergone shear transformations, in which the purple ones are predicted as rearranged. (b) Contour map of $\Delta D_{\min }^{2}$ in a slice of thickness $3.5 \AA$. White spots are ML predicted arranged ones. (c) The probability that an atom is predicted as active versus $\Delta D_{\min }^{2}$.

bution of the atoms that have undergone, or not, plastic rearrangements: among them, purple spheres mark the atoms that are predicted to act as defects by the ML model, while yellow spheres represent the atoms in the glassy matrix which remain inactive during the deformation. It implies that atoms identified as defects by the SVM model are very likely to participate in plastic deformation.

For the testing sample, the prediction accuracy is $78.4 \%$; and the ML model captures $76.4 \%$ of the real 
rearrangements up to global shear strain magnitude of $5 \%$. Fig. 8(b) shows the contour map of the magnitude of the instant jumps in non-affine displacement, $\Delta D_{\min }^{2}$, under AQS deformation. We notice that the distribution of $\Delta D_{\min }^{2}$ is inhomogeneous - atoms with high $\Delta D_{\min }^{2}$ tend to aggregate spatially into clusters, which are responsible for initiating the plastic shear transformations. For a direct comparison, white spots are superimposed to mark the positions of the atoms that are predicted as defects by ML model. The good overlap implies that the spatial distribution of the rearranged atoms is also heterogenous and, interestingly, almost coincides with the regions of high $\Delta D_{\min }^{2}$. This indicates that plastic rearrangements have a high propensity to originate from the plastically-active regions predicted by the ML model. Fig. 8(c) shows the probability of an atom will undergo plastic deformation, which goes up with increasing $\Delta D_{\min }^{2}$ and saturates at a value of approximately 0.87 after $\Delta D_{\min }^{2}=0.1 \AA^{2}$. This is meaningful since the more plastically-active the atom is, the more likely it is to be predicted as glassy defect by the ML model.

\section{Integrated glassy defect}

The value of the integrated glassy defect - IGD that is a parameter assigned for the $i$ th atom - is quantified by the decision value of the ML model. It distinguishes the glassy defects from the inert glassy matrix. This scalar value is proportional to the orthogonal distance between a given position in the input space and the hyperplane constructed by the ML model, with the same scale factor for all atoms. Notably, when IGD $>0$, the corresponding data point in the input space lies on the glassy defect side of the hyperplane; and IGD $<0$ otherwise.

Figure 9 shows the features of the IGD. Fig. 9(a) is a $3 \mathrm{D}$ snapshot with atoms colored according to the magnitude of their IGD parameter value. We notice that the spatial distribution of IGD is heterogeneous with a strong spatial correlation. Fig. 9(b) displays the probability distribution of IGD for the rearranged (red), non-rearranged (blue), and all atoms (black), respectively. There exists a two-peak feature in the total distribution, with the right peak denoting the defect atoms. The rearranging and non-rearranging atoms are separated fairly well in the IGD distribution. Compared with the non-rearranging atoms, the peak position of the rearranging atoms shifts to the right. This indicates that the machine-learned scalar parameter, IGD, is indeed an effective predictor of the atomic plastic rearrangements. It is indeed a physically-relevant measure of the degree of structural defect in glass. Besides, IGD is distributed over a very broad range, indicating strong structural heterogeneity as commonly observed in experimental characterizations [19], which also resembles the inhomogeneous distribution of the non-affine displacements shown in Fig. 3(b). It should be noted that the distribution of IGD is different from that of softness in Ref. [44], the latter shows a symmetric distribution peaked at the origin. The broad distribution of IGD mainly comes from the additional input features beyond "softness". For example, vMSD usually exhibits some distributions with a Gaussian core and non-Gaussian tails [37]. Such non-Gaussian tail is usually the physical origin of the dynamic heterogeneity in glasses $[60,61]$ and is the cause for the broad and asymmetric feature of distribution of IGD.

To measure the universal spatial distribution of IGD, we define a normalized spatial autocorrelation functions, $C(r)=\frac{\left\langle\Delta P_{r_{0}} \Delta P_{r_{0}+r}\right\rangle-\left\langle\Delta P_{r_{0}}\right\rangle^{2}}{\left\langle\Delta P_{r_{0}}{ }^{2}\right\rangle-\left\langle\Delta P_{r_{0}}\right\rangle^{2}}$, where $P$ represents a physical entity. The angle brackets $\langle\cdots\rangle$ denote ensemble average. $P_{r_{0}}$ and $P_{r_{0}+r}$ are the values of $P$ for the central atom at reference position $r_{0}$, and the one at a distance of $r$ from the reference atom, respectively. The operator $\Delta$ denotes the deviation from the ensemble average value. The calculated data for $\Delta D_{\min }^{2}$ and IGD are plotted in Fig. 9(c). They both decay exponentially with respect to $r$. We adopt an empirical function $C(r) \propto \exp (-r / \xi)$ to fit the correlation data, which brings about $\xi$ as the physically spatial correlation length. The spatial correlation lengths for $\Delta D_{\min }^{2}$ and IGD are almost identical, with $\xi_{\Delta D_{\min }^{2}}=3.1 \AA$ And $\xi_{\mathrm{IGD}}=3.2 \AA$, respectively. This supports the validity of the IGD as a signature of plasticity. Both correlation lengths are approximately $1.2 d$, where $d$ is the effective diameter of a single particle. Here $d=2.7$ $\AA$ as determined from the position of the first peak in the RDF, which has been shown in Fig. 1. It indicates that the sweeping scale of IGD is consistent with the rearrangement size. The conclusion is consistent with a previous report from Cubuk et al. [8].

In Fig. 10, we compare the radial distribution functions (RDFs) of two extreme cases - the $10 \%$ atoms with the highest IGD values and those $10 \%$ with the lowest IGD values. It indicates that high-IGD atoms present a lower first peak which simultaneously moves to shorter distance compared with that of the low-IGD atoms. Thus, the formers are alike the liquid atoms with high mobility.

\section{Capacity of IGD in predicting athermal structural excitations}

To get deeper insights into the correlation between IGD and the irreversible atomic rearrangements, we compare the spatial distribution of IGD with the non-affine deformation field in Fig. 11(a). The white bubbles represent the atoms which have undergone shear transformations. The bubble size is proportional to the magnitude of $\Delta D_{\min }^{2}$. It is clearly seen that most of the shear transformations take place in the regions with high values of IGD. The machine-learned IGD thus identifies glassy defect with great propensity to undergo shear transformations. As such, the IGD could be regarded as a quantitative parameter to identify defect in glass, in analogy to the Burgers' vector in crystals. Note that not 

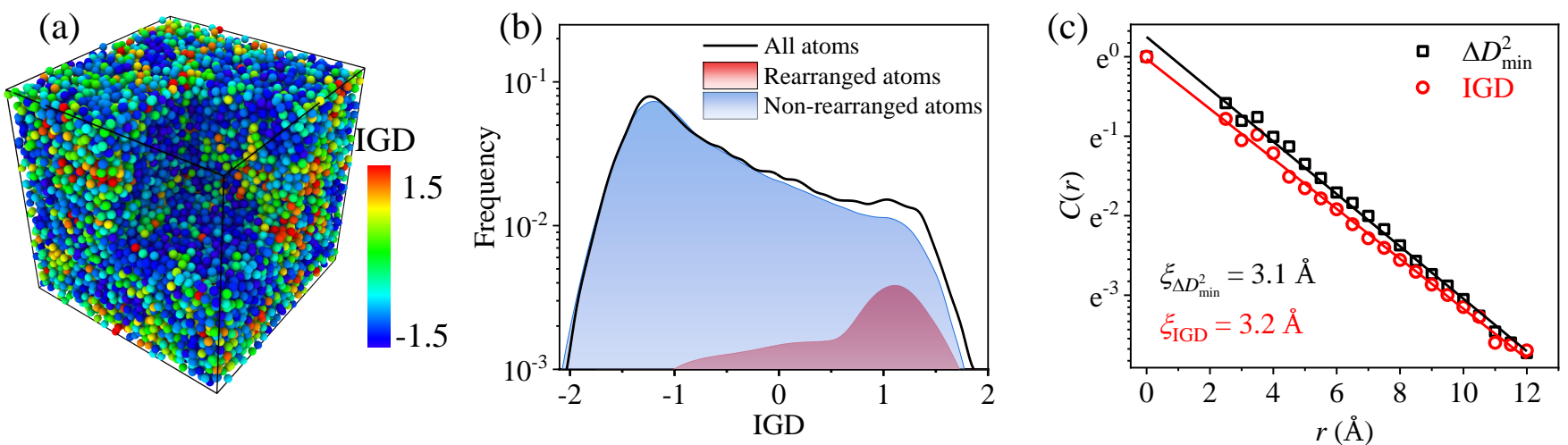

FIG. 9. Features of the integrated glassy defect. (a) A 3D snapshot with particles colored by the magnitude of IGD. A small fraction of the atoms on the cubic corner was removed to see the inner atomic environement. (b) IGD distribution for different atoms. (c) Spatial autocorrelation function versus distance for $\Delta D_{\min }^{2}$ and IGD.
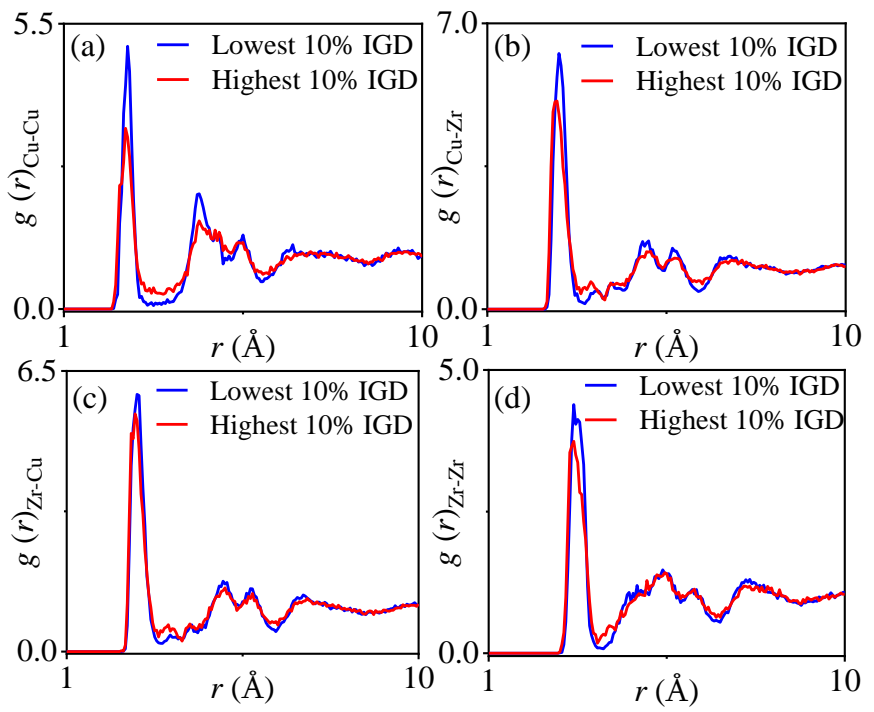

FIG. 10. Partial RDFs for the group of atoms having the lowest $10 \%$ and the highest $10 \%$ IGD in a $\mathrm{Cu}_{50} \mathrm{Zr}_{50}$ metallic glass.

all high-IGD regions should experience plastic rearrangement, which is a stochastic phenomenon and sensitive to the loading protocol and thermal fluctuation $[41,62]$. It is the anisotropic interaction between the glassy defect and the high-dimensional loading direction that cooperatively determines if a glassy defect would lead to real shear transformation. As a result, the rationale for the structure-property relationship in an amorphous system is expected to be statistical. The statistics is shown in Fig. 11(b), with atomic-resolution $\Delta D_{\text {min }}^{2}$ being plotted against IGD. Each data point denotes a coarse graining of $0.25 \%$ of all atoms, with nearly $\sim 50$ atoms in each bin. There exists a strong correlation between IGD and $\Delta D_{\min }^{2}$. The atoms with higher values of IGD contribute more to the non-affine deformation. This result is consistent for both the testing set shown in the main plot, and for the training plus testing set shown in the inset of Fig. 11(b). The agreement once again shows that there exists a robust correlation between the IGD parameter and the plastic deformation in glass.

To demonstrate the efficiency of the integrated strategy in predicting plasticity of glass, we compare IGD with the existing individual physical models/parameters, each taking only one physical aspect (either configurational, thermodynamic, or dynamic) into account. Following the training and prediction protocol as mentioned above, models with only one of $\left\{\mathrm{vMSD}, \Delta Q, G\right.$ and $\left.S_{2}\right\}$ as input descriptors are developed. As shown in Fig. 12, model prediction accuracy for the testing set differs largely with different input descriptors. The predicting accuracy for vMSD is $72.5 \%$, followed by $64.2 \%$ for $\Delta Q, 61.5 \%$ for $G$ and $55.4 \%$ for $S_{2}$, respectively. Thus, the thermodynamic feature is most relevant to structural excitation. Note that the predictor learned from only a family of Gaussian functions is exactly the definition of the "softness" metric [43]. Compared with the L-J model there, the $\mathrm{Cu}-\mathrm{Zr}$ metallic glass described by the many-body EAM potential sets up an upper bound of difficulty for prediction via machine learning. The improved predictability demonstrated by IGD stresses the necessity of including extra thermodynamic and dynamic input for predicting the physical properties of generic, real and complex structural materials.

It should be noted that the prediction accuracy here is not a superior indicator to evaluate the prediction power of plasticity of our simulation results. As there is only $5 \%$ of atoms in the testing set that are labeled as plastic ones, even an unintelligent model which predicts all atoms as non-rearranged ones will achieve 95\% prediction accuracy in the testing set. To further quantify the predictive capability of metrics in assessing glassy plasticity, a power factor is further introduced as $\chi=\frac{P_{R}\left(X_{i}>X_{\text {top } 5 \%}\right)}{P_{R}\left(X_{i}<X_{\text {low } 5 \%}\right)}$, as there is nearly $5 \%$ of all atoms are recognized as rearranged ones in our simulations. Here, $X_{i}$ is the magnitude of the feature $X$ for the particle 
(a)
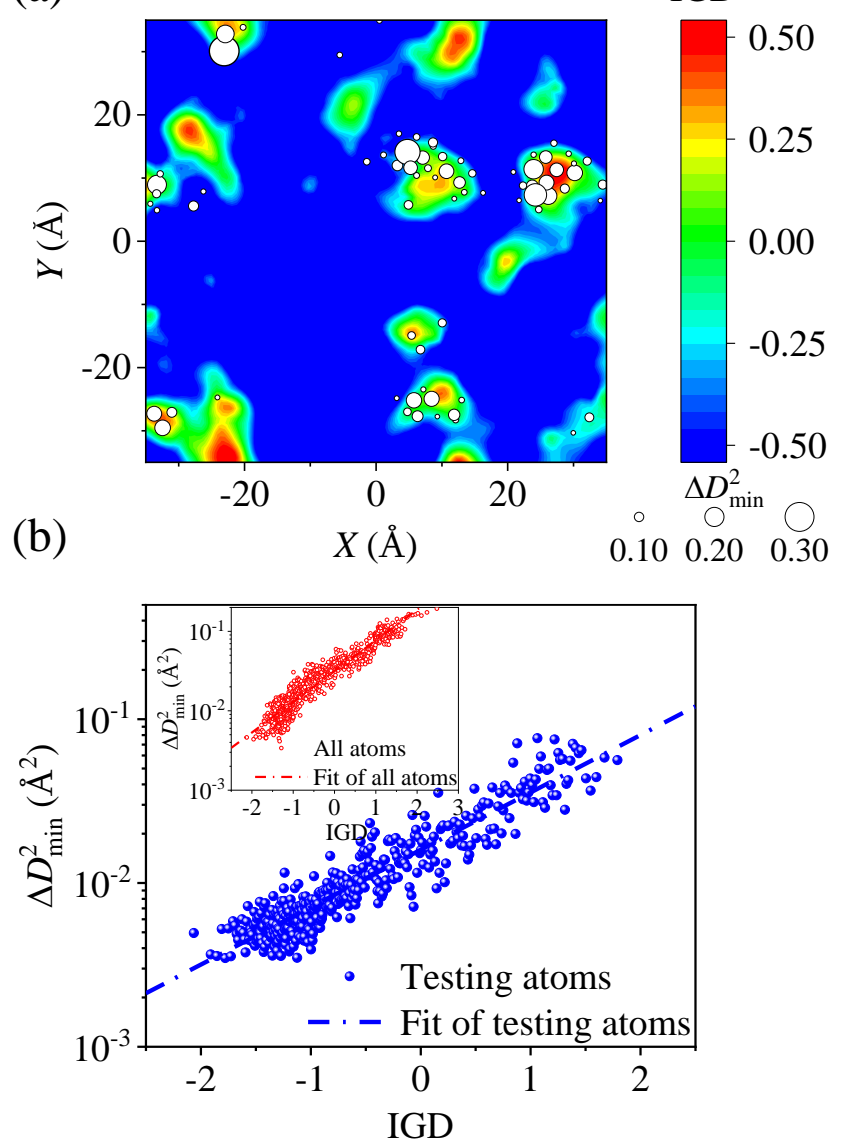

FIG. 11. Capacity of IGD in predicting athermal shear transformations. (a) Contour map of IGD. White bubbles indicate the location of events, and the bubble size is proportional to $\Delta D_{\min }^{2}$. (b) Correlation between IGD and $\Delta D_{\min }^{2}$ in the testing set. Each data point represents an average value of $0.25 \%$ of all atoms. The inset includes both training and testing set.

i. $P_{R}\left(X_{i}>X_{\mathrm{top} 5 \%}\right)$ denotes the probability that a particle rearranges with $X_{i}$ higher than the top $5 \%$ of all atoms, and $P_{R}\left(X_{i}<X_{\text {low5\% }}\right)$ otherwise. Thus the predictive power factor is the ratio of the probability of rearrangements for the atoms with highest $X_{i}$ with respect to that for atoms with the lowest $X_{i}$. It should be noted that this equation only suits for parameters $P_{R}\left(X_{i}\right)$ that monotonically increase with $X_{i}$. When $P_{R}\left(X_{i}\right)$ and $X_{i}$ are inversely proportional or anti-correlated, $\chi$ is replaced by its reciprocal. The higher the magnitude of $\chi$, the better the predictor $X$ in discriminating between plasticallyactive and inactive atoms.

The predictive power factor of different ML models is calculated and plotted in Fig. 12(b), where IGD shows the highest bar. It shows that IGD is superior to any other physical model in predicting plasticity. Even if the basic predictors are of poor predictive power when considered separately, once they are all integrated into IGD via the present ML protocol, the predictive powder does improve drastically. This observation implies that the (a)

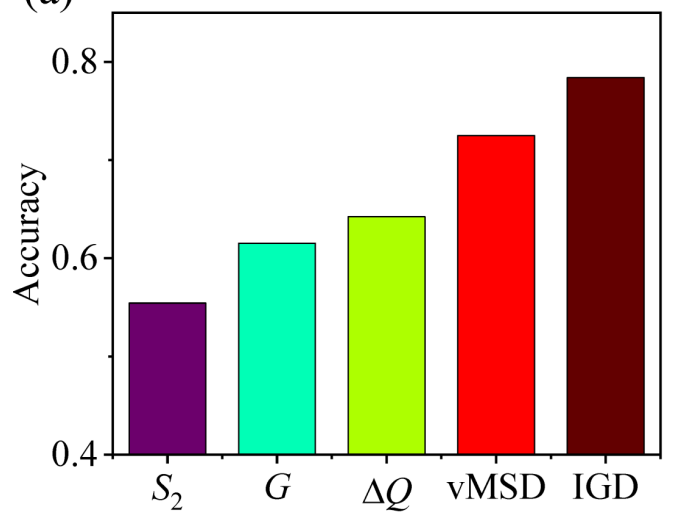

(b)

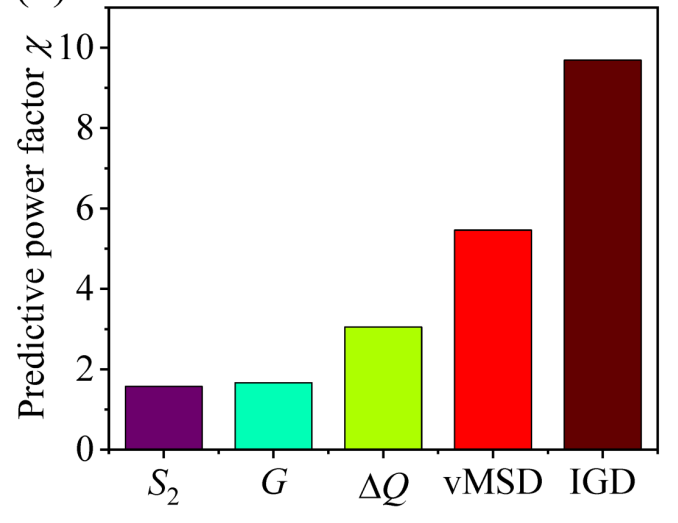

FIG. 12. (a) The prediction accuracy of models with different input descriptors: excess entropy $S_{2, i}$, Gaussian structural function $G_{i}$, activation energy $\Delta Q_{i}$ and vibrational meansquared displacements vMSD. (b) Absolute value of the predictive power factor for IGD in comparison with the other four physical descriptors.

structural, thermodynamic and dynamic descriptors are neither orthogonal, nor absolutely correlated with each other. If they were absolutely correlated, there would be no room to improve the prediction power via integration and, thus, there would be no synergy when they are combined together. We also noticed that vMSD and $\Delta Q$ serve as the most important descriptors in the machinelearning process, while models with only structural features, i.e. $G$ or $S_{2}$, represent poor performance in predicting plasticity. It demonstrates the necessity of including extra thermodynamic and dynamic features as done in our ML process. The inclusion of the thermodynamic and dynamic components not only enhances the classification (since the prediction accuracy of ML model with all of vMSD, $\Delta Q, G$ and $S_{2}$ is nearly $80 \%$, as opposed to $\sim 60 \%$ accuracy achieved by the scheme with only structural features), but also improves the predictive capability of plasticity in metallic glasses.

Our interpretation to this argument is in term of the spatial autocorrelation of the SVM models with different input descriptors, as shown in Fig. 13. It implies 

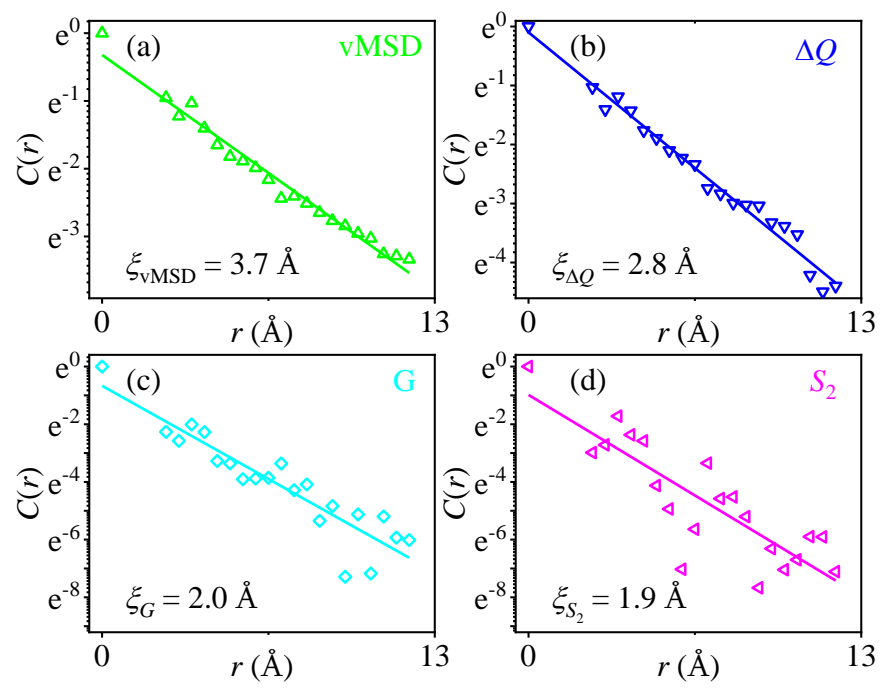

FIG. 13. Semi-logarithmic plot of the spatial autocorrelation function versus distance for the predictors learned from (a) vMSD, (b) $\Delta Q$, (c) $G$ and $S_{2}$, respectively. (d) The dashed lines are the best fits according to the empirical equation $C(r) \propto \exp (-r / \xi)$, defining correlation length $\xi$.

that predictors learned from structural features decreases very quickly with increasing distance. Indeed, it exhibits a decay length of only $1.9 \AA$ for $S_{2}$ and $2.0 \AA$ for $G$, which is much shorter than the size of atom rearrangement - 3.1 $\AA$ as shown Fig. 9(c). This is presumably a possible explanation for the poor predictive capability of the purely structural function, which lacks information about medium- to long-range interactions. Besides, atomic structures vibrate under the influence of thermal fluctuations, therefore the definition of glassy defect from static structure might be not unique. In a real thermal glass, the relationship between structure and property is rather complex such that unstable phonon saddles are important, as evidenced by the instantaneous normal mode analysis of the mechanical stability [63]. This is also the reason why the flexibility volume [37] instead of Voronoi volume correlates strongly with structural excitation owing to the fact that the former has crucially incorporated the thermodynamic Debye-Waller factor.

\section{E. Transferability of IGD}

The remaining question is whether the IGD - learned from supervised machine learning with athermal mechanical excitation as supervisory signal - can be transferred to predict also the thermal (or dynamic) properties of glasses. The latter properties are important for many applications and for routine material characterization. We test the IGD by correlating it with several important short-time, and long-time properties that have not been considered at all in the ML protocol thus far. The dynamic properties include the vibrational boson peak, diffusion heterogeneity and the $\alpha$ relaxation, which are properties that span multiple time scales.

\section{Correlating IGD with boson peak}

First of all, we begin with the correlation between IGD and boson peak, the latter of which is a universal shorttime vibrational anomaly - with extra soft modes over the Debye squared law - in a wide range of amorphous solids [24, 64]. For this purpose, we calculate an atomistically resolved boson peak intensity in terms of the partial vibrational mode anomaly at atomic scale. The intensity of boson peak is represented by the peak value of the reduced vibrational density of states [VDOS, $g(\omega)$ ] for a single atom. As shown in the section methodology, the normal mode analysis is performed by directly diagonalizing the dynamical matrix (or Hessian matrix) of the system. Once the whole vibrational spectra are available, they are projected onto a single atom $i$, i.e. $g_{i}(\omega)$. Since the Debye model gives a quadratic distribution of the normal-mode frequency $-g(\omega) \propto \omega^{2}$, the single-particle intensity of boson peak can be derived as the height of the peak in the reduced VDOS over the Debye model $I_{\mathrm{BP}}=\max \left[g_{i}(\omega) / \omega^{2}\right]$.

Figure 14(a) displays the reduced VDOS, $g_{i}(\omega) / \omega^{2}$, for the atoms with different mean values of IGD. As seen in Fig. 14(a), atoms with different values of IGD display different intensity of boson peaks. These boson peaks are arranged from the weakest to the strongest as IGD increases. It indicates that defects characterized by high value of IGD have lower tendency to participate in the high-frequency vibrations, thus giving rise to a relatively high intensity of boson peak at lower frequency. To further confirm the correlation, we directly plot the intensity of boson peak, $I_{\mathrm{BP}}$, versus IGD using a bin size of 500 atoms, which is nearly equal to $2.5 \%$ of all atoms in the testing sample. As shown in Fig. 14(b), a good linear correlation exists between IGD and $I_{\mathrm{BP}}$. Therefore, IGD can be also used to predict the boson peak in glasses, with even better predictability than our earlier proposed purely structural metric - the short-range orientational order parameter defined as the longest vector from a central atom to its Voronoi polyhedron vertex [24].

\section{Correlating IGD with diffusion heterogeneity}

We can now consider how the IGD parameter correlates with the long-time diffusion dynamics. Here, the diffusive MSD is utilized to benchmark a direct link between IGD and the slow dynamics. Fig. 14(c) depicts the diffusive MSD at $700 \mathrm{~K}$ for the atoms with different values of IGD. Obviously, the atoms with the highest IGD are these ones first escape from the caging plateau. In other words, they are the most active atoms that contribute to the diffusion dynamics. At the same time, the slowing down of dynamics for the low-IGD atoms 

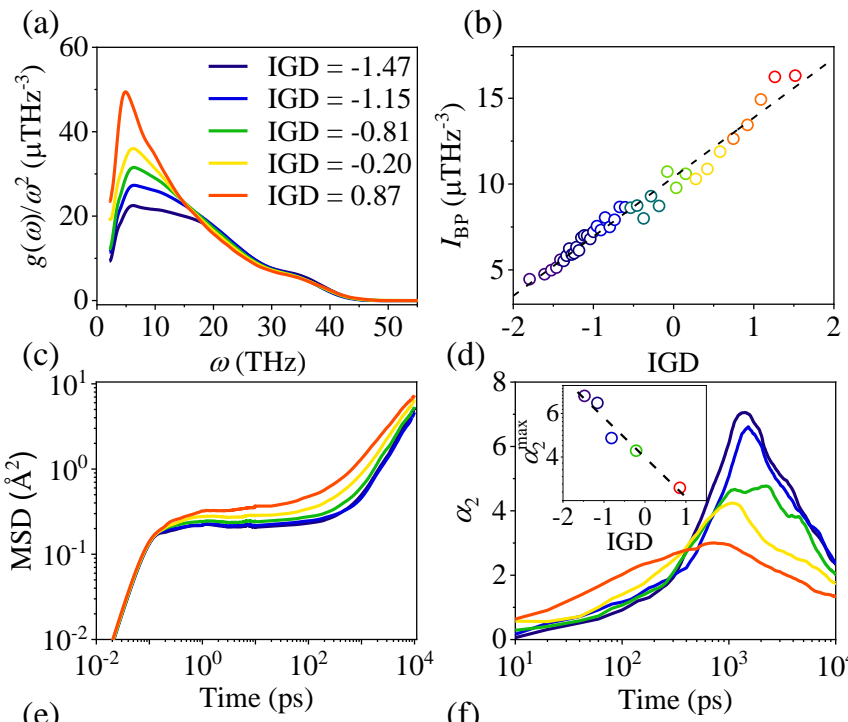

(e)

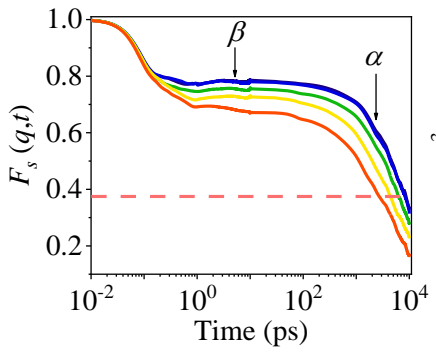

(f)
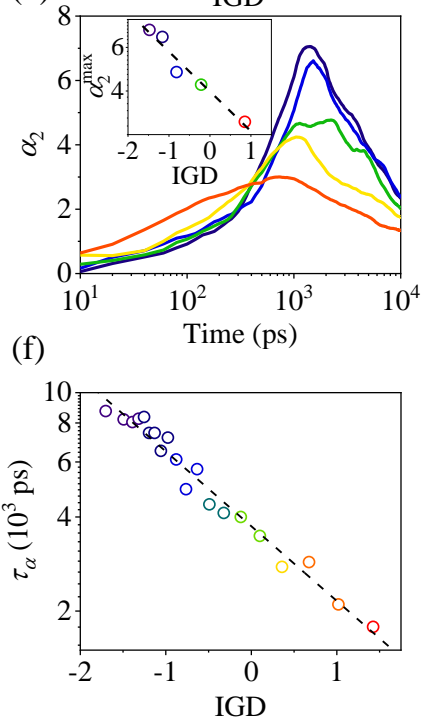

FIG. 14. Transferability of IGD in predicting dynamic properties of glass. (a) Reduced VDOS for the atoms with different values of IGD. (b) Correlation between the boson peak intensity and IGD. Each data point denotes the average value of $2.5 \%$ of all atoms. (c) Long-time diffusive MSD of atoms with different values of IGD at $700 \mathrm{~K}$. (d) Non-Gaussian parameter corresponding to (c). Inset: $\alpha_{2}^{\max }$ versus IGD. (e) Self-intermediate scattering function of atoms with different IGD. (f) $\alpha$-relaxation time as a function of IGD.

is accompanied by an increasing dynamic heterogeneity, which is signaled by the rise of the non-Gaussian parameter $\alpha_{2}$ with decreasing IGD, as shown in Fig. 14(d). Generally, the peak value of the non-Gaussian parameter, $\alpha_{2}^{\max }$, can be used to quantify the degree of dynamic heterogeneity. As seen in the inset of Fig. 14(d), we indeed find a strongly inverse correlation between the value of IGD and the magnitude of $\alpha_{2}^{\max }$. It actually reveals the liquid-like nature of the atoms with high value of IGD, and the solid-like nature of the low-IGD atoms. This is in agreement with the common sense that dynamic heterogeneity of a disordered material diminishes with increasing temperature upon crossing the glass-to-liquid transition [65, 66]. Finally, $\alpha_{2}^{\max }$ of the low-IGD atoms rises on a longer time-scale. This phenomenon means that the low-IGD atoms are in relatively deep glassy state, which takes a longer time to escape from the relatively deep basin of the $\beta$ relaxation on the PEL.

\section{Correlating IGD with the $\alpha$-relaxation}

The other long-time dynamic feature to be examined is the slow relaxation behavior. To this end, we investigate the self-intermediate scattering function [SISF, $F_{\mathrm{S}}(\mathbf{q}, t)$ ] of the atoms with different values of IGD. The results are shown in Fig. 14(e). It is evident that the relaxation dynamics slows down dramatically with decreasing value of IGD, represented by an extended plateau on the SISF curve, which corresponds to the secondary $\beta$-relaxation process. This trend indicates that motion of the low-IGD atoms is highly confined by the neighboring atoms, displaying a significant cage effect [67]. The revealed scenario for relaxation is consistent with that of diffusive MSD shown previously in Fig. 14(c), as both of them have the same origin in the local basin climbing process on the PEL.

In general, the characteristic timescale of the relaxation dynamics can be quantified by the $\alpha$-relaxation time $\tau_{\alpha}$, which is defined as the time where SISF has decayed to $e^{-1}$, as marked by the horizontal dashed line in Fig. 14(e). The $\alpha$-relaxation times for those atoms with different value of IGD are shown in Fig. 14(f). A very strong correlation is revealed between IGD and the $\alpha$-relaxation time. Thus, one can treat IGD as an effective indicator of the relaxation propensity at atomic scale.

In short sum, the newly developed IGD parameter proves highly versatile in being able to predict the shorttime boson peak vibrational phenomenon, the longtime thermally-activated diffusion and the $\alpha$-relaxation dynamics. Surprisingly, these dynamic features have never been considered in the supervised machine learning model in which only athermal shear transformation is implemented as the supervisory signal. In this sense, the proposed concept of integrated glassy defect can serve as a generalized "super-defect", in analogy to the conventional crystalline imperfections - like vacancies, dislocations, grain boundaries etc - to identify the carriers of plasticity in amorphous solids [14].

\section{CONCLUDING DISCUSSION}

In conclusion, we identified a "super-defect" - termed IGD - to build possible structure-property relationship for the disordered materials by using extensive machine learning classification from a wide, intricate and partially entangled configurational-thermodynamic-dynamic space of data. IGD is glassy defect in the sense that the atom with high value of IGD has the highest propensity to undergo plastic shear transformation under athermal mechanical perturbations. As a step beyond the usual purely static structural descriptors, the present machinelearned IGD incorporates also thermodynamic and dynamical features characterized by the vibrational excitations and the thermal-activation events from probing the morphology of local potential energy landscape. Infor- 
mation from a single physical input can only partially capture the nature of the plastic deformation. In the case of complex media, it is the synergistic cooperation of both structural and thermodynamic-dynamic fingerprints that jointly determines the properties.

The integrated defect also allows one to disentangle the respective roles of different sectors of the overall phase space in governing the properties of glasses. Amongst the versatile descriptors is the thermodynamic feature that is crucial for predicting (athermal) structural excitation in glasses, and also provides a physical foundation for the successes of the vibrational amplitude and the flexibility volume as effective signatures of glass dynamics. In addition to the plastic deformation, the IGD is also shown to be a quantitative metric for other important and general thermal properties, such as the short-time low-frequency vibrational anomaly - the intensity of boson peak, as well as the long-time $\alpha$-relaxation and diffusion heterogeneity in amorphous alloys.

Our method relies on static structure, thermodynamics as well as dynamics of a group of atoms. Thus, the methodology is applicable directly to other glass systems, where Gaussian functions, two-body excess entropy, vibrational mean-squared displacement as well as activation energies can be calculated at atomic scale. The "softness" with only Gaussian functions as input has been tested vastly in different systems, such LennardJones (LJ) mixture, Oligomer as well as Granular materials, and all shows acceptable predicting capacity $[8,43]$. Here, the present strategy suggests that the combination of configurational-thermodynamic-dynamic features can construct comprehensive atomic environment and yield a powerful predictability of IGD. Thus, one can reliably infer that the IGD will perform as well for other disordered systems since the real-atom $\mathrm{CuZr}$ system always represents an upper bound of prediction for the property of glasses [48].

The proposed concept of IGD opens up new opportunities to optimize the physical properties of disordered materials by modulating the material processing conditions, besides the straightforward chemical-design of material structure.

\section{ACKNOWLEDGMENTS}

This work is financially supported by the National Key Research and Development Program of China (No. 2017YFB0701502), the National Natural Science Foundation of China (No. 12072344), and the Youth Innovation Promotion Association of Chinese Academy of Sciences (No. 2017025).
[1] A. L. Greer, Metallic Glasses, Science 267, 1947 (1995).

[2] C. A. Schuh and A. C. Lund, Atomistic basis for the plastic yield criterion of metallic glass, Nat. Mater. 2, 449 (2003).

[3] P. Schall, D. A. Weitz, and F. Spaepen, Structural Rearrangements That Govern Flow in Colloidal Glasses, Science 318, 1895 (2007).

[4] A. Furukawa and H. Tanaka, Inhomogeneous flow and fracture of glassymaterials, Nat. Mater. 8, 601 (2009).

[5] E. Ma, Tuning order in disorder, Nat. Mater. 14, 547 (2015).

[6] C. A. Schuh, T. C. Hufnagel, and U. Ramamurty, Mechanical behavior of amorphous alloys, Acta Mater. 55, 4067 (2007).

[7] Y. Q. Cheng and E. Ma, Atomic-level structure and structure-property relationship in metallic glasses, Prog. Mater. Sci. 56, 379 (2011).

[8] E. D. Cubuk, R. J. S. Ivancic, S. S. Schoenholz, D. J. Strickland, A. Basu, Z. S. Davidson, J. Fontaine, J. L. Hor, Y.-R. Huang, Y. Jiang, N. C. Keim, K. D. Koshigan, J. A. Lefever, T. Liu, X.-G. Ma, D. J. Magagnosc, E. Morrow, C. P. Ortiz, J. M. Rieser, A. Shavit, T. Still, Y. Xu, Y. Zhang, K. N. Nordstrom, P. E. Arratia, R. W. Carpick, D. J. Durian, Z. Fakhraai, D. J. Jerolmack, D. Lee, J. Li, R. Riggleman, K. T. Turner, A. G. Yodh, D. S. Gianola, and A. J. Liu, Structure-property relationships from universal signatures of plasticity in disordered solids, Science 358, 1033 (2017).

[9] P. G. Debenedetti and F. H. Stillinger, Supercooled liquids and the glass transition, Nature 410, 259 (2001).

[10] V. Lubchenko and P. G. Wolynes, Theory of Struc- tural Glasses and Supercooled Liquids, Annu. Rev. Phys. Chem. 58, 235 (2007).

[11] Y. Fan, T. Iwashita, and T. Egami, How thermally activated deformation starts in metallic glass, Nat. Commun. 5, 5083 (2014).

[12] Y.-C. Hu, Y.-W. Li, Y. Yang, P.-F. Guan, H.-Y. Bai, and W.-H. Wang, Configuration correlation governs slow dynamics of supercooled metallic liquids, Proc. Natl. Acad. Sci. U. S. A. 115, 6375 (2018).

[13] H. Tanaka, H. Tong, R. Shi, and J. Russo, Revealing key structural features hidden in liquids and glasses, Nat. Rev. Phys. 1, 333 (2019).

[14] W. Cai and W. D. Nix, Imperfections in crystalline solids (Cambridge University Press, 2016).

[15] F. Spaepen, A microscopic mechanism for steady state inhomogeneous flow in metallic glasses, Acta Metall. 25, 407 (1977).

[16] A. S. Argon, Plastic deformation in metallic glasses, Acta Metall. 27, 47 (1979).

[17] M. L. Falk and J. S. Langer, Dynamics of viscoplastic deformation in amorphous solids, Phys. Rev. E 57, 7192 (1998).

[18] W. L. Johnson and K. Samwer, A Universal Criterion for Plastic Yielding of Metallic Glasses with a $\left(T / T_{g}\right)^{(2 / 3)}$ Temperature Dependence, Phys. Rev. Lett. 95, 195501 (2005).

[19] J. C. Qiao, Q. Wang, J. M. Pelletier, H. Kato, R. Casalini, D. Crespo, E. Pineda, Y. Yao, and Y. Yang, Structural heterogeneities and mechanical behavior of amorphous alloys, Prog. Mater. Sci. 104, 250 (2019).

[20] H. W. Sheng, W. K. Luo, F. M. Alamgir, J. M. Bai, and 
E. Ma, Atomic packing and short-to-medium-range order in metallic glasses, Nature 439, 419 (2006).

[21] Y. C. Hu, F. X. Li, M. Z. Li, H. Y. Bai, and W. H. Wang, Five-fold symmetry as indicator of dynamic arrest in metallic glass-forming liquids, Nat. Commun. 6, 9310 (2015).

[22] H. L. Peng, M. Z. Li, and W. H. Wang, Structural Signature of Plastic Deformation in Metallic Glasses, Phys. Rev. Lett. 106, 135503 (2011).

[23] J. M. Rieser, C. P. Goodrich, A. J. Liu, and D. J. Durian, Divergence of Voronoi Cell Anisotropy Vector: A Threshold-Free Characterization of Local Structure in Amorphous Materials, Phys. Rev. Lett. 116, 088001 (2016).

[24] J. Yang, Y.-J. Wang, E. Ma, A. Zaccone, L. H. Dai, and M. Q. Jiang, Structural Parameter of Orientational Order to Predict the Boson Vibrational Anomaly in Glasses, Phys. Rev. Lett. 122, 015501 (2019).

[25] R. Milkus and A. Zaccone, Local inversion-symmetry breaking controls the boson peak in glasses and crystals, Phys. Rev. B 93, 094204 (2016).

[26] D. C. Wallace, On the role of density fluctuations in the entropy of a fluid, J. Chem. Phys. 87, 2282 (1987).

[27] X. Yang, R. Liu, M. Yang, W.-H. Wang, and K. Chen, Structures of Local Rearrangements in Soft Colloidal Glasses, Phys. Rev. Lett. 116, 238003 (2016).

[28] M. Laurati, P. Maßhoff, K. J. Mutch, S. U. Egelhaaf, and A. Zaccone, Long-lived neighbors determine the rheological response of glasses, Phys. Rev. Lett. 118, 018002 (2017).

[29] H. Tong and H. Tanaka, Revealing Hidden Structural Order Controlling Both Fast and Slow Glassy Dynamics in Supercooled Liquids, Phys. Rev. X 8, 011041 (2018).

[30] H. Tong and H. Tanaka, Structural order as a genuine control parameter of dynamics in simple glass formers, Nat. Commun. 10, 5596 (2019).

[31] H. Tong and H. Tanaka, Role of Attractive Interactions in Structure Ordering and Dynamics of Glass-Forming Liquids, Phys. Rev. Lett. 124, 225501 (2020).

[32] A. Widmer-Cooper, H. Perry, P. Harrowell, and D. R. Reichman, Irreversible reorganization in a supercooled liquid originates from localized soft modes, Nat. Phys. 4, 711 (2008).

[33] A. Widmer-Cooper, H. Perry, P. Harrowell, and D. R. Reichman, Localized soft modes and the supercooled liquid's irreversible passage through its configuration space, J. Chem. Phys. 131, 194508 (2009).

[34] J. Ding, S. Patinet, M. L. Falk, Y. Cheng, and E. Ma, Soft spots and their structural signature in a metallic glass, Proc. Natl. Acad. Sci. 111, 14052 (2014).

[35] A. Widmer-Cooper, P. Harrowell, and H. Fynewever, How Reproducible Are Dynamic Heterogeneities in a Supercooled Liquid?, Phys. Rev. Lett. 93, 135701 (2004).

[36] L. Larini, A. Ottochian, C. De Michele, and D. Leporini, Universal scaling between structural relaxation and vibrational dynamics inglass-forming liquids and polymers, Nat. Phys. 4, 42 (2008).

[37] J. Ding, Y.-Q. Cheng, H. Sheng, M. Asta, R. O. Ritchie, and E. Ma, Universal structural parameter to quantitatively predict metallic glass properties, Nat. Commun. 7, 13733 (2016).

[38] Z. Fan, J. Ding, Q.-J. Li, and E. Ma, Correlating the properties of amorphous silicon with its flexibility volume, Phys. Rev. B 95, 144211 (2017).
[39] J. Zylberg, E. Lerner, Y. Bar-Sinai, E. Bouchbinder, and J. S. Langer, Local thermal energy as a structural indicator in glasses, Proc. Natl. Acad. Sci. 114, 7289 (2017).

[40] S. Patinet, D. Vandembroucq, and M. L. Falk, Connecting Local Yield Stresses with Plastic Activity in Amorphous Solids, Phys. Rev. Lett. 117, 045501 (2016).

[41] B. Xu, M. L. Falk, J. F. Li, and L. T. Kong, Predicting Shear Transformation Events in Metallic Glasses, Phys. Rev. Lett. 120, 125503 (2018).

[42] J. Behler and M. Parrinello, Generalized Neural-Network Representation of High-Dimensional Potential-Energy Surfaces, Phys. Rev. Lett. 98, 146401 (2007).

[43] E. D. Cubuk, S. S. Schoenholz, J. M. Rieser, B. D. Malone, J. Rottler, D. J. Durian, E. Kaxiras, and A. J. Liu, Identifying structural flow defects in disordered solids using machine-learning methods, Phys. Rev. Lett. 114, 108001 (2015).

[44] S. S. Schoenholz, E. D. Cubuk, D. M. Sussman, E. Kaxiras, and A. J. Liu, A structural approach to relaxation in glassy liquids, Nat. Phys. 12, 469 (2016).

[45] Q. Wang and A. Jain, A transferable machine-learning framework linking interstice distribution and plastic heterogeneity in metallic glasses, Nat. Commun. 10, 5537 (2019).

[46] R. Freitas and E. J. Reed, Uncovering the effects of interface-induced ordering of liquid on crystal growth using machine learning, Nat. Commun. 11, 3260 (2020).

[47] V. Bapst, T. Keck, A. Grabska-Barwińska, C. Donner, E. D. Cubuk, S. S. Schoenholz, A. Obika, A. W. R. Nelson, T. Back, D. Hassabis, and P. Kohli, Unveiling the predictive power of static structure in glassy systems, Nat. Phys. 16, 448 (2020).

[48] Z. Fan, J. Ding, and E. Ma, Machine learning bridges local static structure with multiple properties in metallic glasses, Mater. Today 40, 48 (2020).

[49] S. Plimpton, Fast parallel algorithms for short-range molecular dynamics, J. Comput. Phys. 117, 1 (1995).

[50] M. I. Mendelev, M. J. Kramer, R. T. Ott, and D. J. Sordelet, Molecular dynamics simulation of diffusion in supercooled Cu-Zr alloys, Philos. Mag. 89, 109 (2009).

[51] S. Nosé, A unified formulation of the constant temperature molecular dynamics methods, J. Chem. Phys. 81, 511 (1984).

[52] M. Parrinello and A. Rahman, Polymorphic transitions in single crystals: A new molecular dynamics method, J. Appl. Phys. 52, 7182 (1981).

[53] C. E. Maloney and A. Lemaître, Amorphous systems in athermal, quasistatic shear, Phys. Rev. E 74, 016118 (2006).

[54] T. S. Ingebrigtsen and H. Tanaka, Structural predictor for nonlinear sheared dynamics in simple glass-forming liquids, Proc. Natl. Acad. Sci. U. S. A. 115, 87 (2018).

[55] G. T. Barkema and N. Mousseau, Event-based relaxation of continuous disordered systems, Phys. Rev. Lett. 77, 4358 (1996).

[56] R. Malek and N. Mousseau, Dynamics of Lennard-Jones clusters: A characterization of the activation-relaxation technique, Phys. Rev. E 62, 7723 (2000).

[57] C.-C. Chang and C.-J. Lin, LIBSVM, ACM Trans. Intell. Syst. Technol. 2, 1 (2011).

[58] A. Togo and I. Tanaka, First principles phonon calculations in materials science, Scr. Mater. 108, 1 (2015).

[59] Q. Wang, J. Ding, L. Zhang, E. Podryabinkin, A. Shapeev, and E. Ma, Predicting the propensity for 
thermally activated $\beta$ events in metallic glasses via interpretable machine learning, npj Comput. Mater. 6, 194 (2020), 2006.13552.

[60] P. Chaudhuri, L. Berthier, and W. Kob, Universal Nature of Particle Displacements close to Glass and Jamming Transitions, Phys. Rev. Lett. 99, 060604 (2007).

[61] P. Cao, M. P. Short, and S. Yip, Potential energy landscape activations governing plastic flows in glass rheology, Proc. Natl. Acad. Sci. 116, 18790 (2019).

[62] Z. Fan and E. Ma, Predicting orientation-dependent plastic susceptibility from static structure in amorphous solids via deep learning, Nat. Commun. 12, 1506 (2021).

[63] A. Zaccone and M. Baggioli, Universal law for the vibrational density of states of liquids, Proc. Natl. Acad. Sci. U. S. A. 118, 2 (2021).
[64] M. Baggioli and A. Zaccone, Universal Origin of Boson Peak Vibrational Anomalies in Ordered Crystals and in Amorphous Materials, Phys. Rev. Lett. 122, 145501 (2019).

[65] Z. Wang, B. A. Sun, H. Y. Bai, and W. H. Wang, Evolution of hidden localized flow during glass-to-liquid transition in metallic glass, Nat. Commun. 5, 5823 (2014).

[66] D. Han, D. Wei, P.-H. Cao, Y.-J. Wang, and L.-H. Dai, Statistical complexity of potential energy landscape as a dynamic signature of the glass transition, Phys. Rev. B 101, 064205 (2020).

[67] B. Li, K. Lou, W. Kob, and S. Granick, Anatomy of cage formation in a two-dimensional glass-forming liquid, Nature 587, 225 (2020). 